\title{
Auftragschweißen von nanokristallin erstarrenden Eisenbasiswerkstoffen auf Aluminiumsubstraten
}

\section{Napawanie nanostrukturalnie krystalizujących materiałów na osnowie żelaza na podłoże aluminium}

\section{Streszczenie}

Przeanalizowano metody zmniejszenia wprowadzenia ilości ciepła do napawanego podłoża. Wykazano korzystne właściwości napoin o budowie nanostrukturalnej. Przedstawiono wyniki badań strukturalnych napoin z zastosowaniem drutów proszkowych (stopy na osnowie żelaza) na stop aluminium AISi8Cu3, przy uzyskaniu niskoenergetycznych warstw napawania, w tym z zastosowaniem warstw przejściowych.

\section{Abstract}

The methods of the heat input to the surfacing by welding substrate decrease is analyzed. It is shown the good properties of nanostructure padding welds. Structure analysis of padding welds deposited with the use of flux wire (Fe matrix alloys) on the AISi8Cu3 aluminum alloy substrate is presented. The low energy and transient pad weIded layers are reached.

\section{Einleitung}

Die zunehmenden Anforderungen an Fahrzeuge und andere Konstruktionen bezüglich ihrer Unterhaltskosten, Umweltverträglichkeit und Leistungsdichte zwingen die Hersteller vermehrt zu Leichtbaukonstruktionen. Somit lässt sich beispielsweise über Gewichtseinsparungen der Kraftstoffverbrauch senken und die Fahrdynamik verbessern. Neben neuartigen hochfesten Stahlwerkstoffen kommen vor allem Aluminiumlegierungen zum Einsatz. Aluminiumguss- bzw. Knetwerkstoffe sind seit vielen Jahren als Werkstoffe für den konstruktiven Leichtbau etabliert und werden heute sehr umfangreich eingesetzt. Gilt es, an derartigen Bauteilen einen partiellen Verschleißschutz zum Beispiel für Dichtflächen wie beispielsweise Ventilsitze in Kompressoren, Motoren, Armaturen oder auch Lagersitze in Getrieben oder Querlenkern zu realisieren, so kommen heute häufig Inlays aus einem verschleißbeständigen Werkstoff zur Anwendung, da Aluminium-

Prof. Dr.-Ing. habil. Johannes Wilden - Funktionswerkstoffe und Beschichtungen Hochschule Niederrhein, Niemcy. legierungen im Allgemeinen nur einen geringen Verschleißschutz gewährleisten. Hierfür ist einerseits bereits bei der Konstruktion Bauraum vorzusehen und andererseits der Fertigungsaufwand, beispielsweise für Passungen, sehr hoch. Um den sicheren Sitz von Inlays zu gewährleisten, muss sowohl der Sitz im Aluminium als auch das Inlay mit hoher Genauigkeit gefertigt werden, das Inlay eingepresst oder eingeschrumpft und anschließend die Dichtfläche entsprechend den geforderten Form- und Lagetoleranzen sowie der Oberflächenqualität endbearbeitet werden. Auch im Betrieb ist diese Konstruktion nicht unproblematisch, da sich beispielsweise bei Thermozyklen oder auch im Dauereinsatz bei erhöhter Temperatur infolge der unterschiedlichen Ausdehnungskoeffizienten das Inlay lockern kann, was u.a. auch in Folge eines veränderten Wärmestromes zum Versagen des Dichtsitzes führen kann.

Eine ideale Lösung wäre das Auftragschweißen beispielsweise des Dichtrings aus einer verschleißbeständigen Legierung z.B. auf Eisenbasis. Für die technische Umsetzung muss dabei sowohl die Problematik der Bildung intermetallischer Phasen im Interface und die Größe des Schmelzbades während des Beschichtungsvorganges, als auch die Problematik während des Betriebs auftretender thermischer Spannungen 
im Interface zwischen Al-Legierung und Beschichtung beherrscht werden.

Die geregelte Kurzlichtbogentechnik, welche in den letzten Jahren bis zur Marktreife entwickelt worden ist, bietet die Möglichkeit, kostengünstig und sowohl automatisiert, teilmechanisiert als auch händisch Auftragschweißungen herzustellen. Aufgrund des kontrollierten Wärmeeintrages besitzt diese Technologie das Potenzial, die Bildung intermetallischer Phasen zu begrenzen und die Schmelzbadgröße einzuschränken.

Als Beschichtungswerkstoffe eröffnen innovative, nanokristallin erstarrende Eisenbasislegierungen völlig neue Eigenschaftsprofile. Neben hoher Verschleißbeständigkeit können diese je nach Gefügestruktur extrem geringe Wärmeleitfähigkeiten aufweisen, wie sie bisher von keramischen Wärmedämmschichten bekannt sind.

In dem Forschungsvorhaben sollen die prozesstechnischen mit den werkstofftechnischen Möglichkeiten zu einer Technologie zum Beschichten von Aluminiumlegierungen mit nanokristallin erstarrenden Eisenbasiswerkstoffen zusammengeführt werden.

\section{Stand der Technik}

Das Ausnutzen nanoskaliger Effekte bietet vielfältige über den derzeitigen Stand der Technik hinausgehende Möglichkeiten, Werkstoffeigenschaften auch auf sich scheinbar widersprechende Anforderungen auszulegen. Die technische Umsetzung derartiger Möglichkeiten erfolgt in der „klassischen“ Beschichtungstechnik bisher nicht, so dass dieses Potenzial ungenutzt bleibt. Jüngste Entwicklungen in der Werkstofftechnologie haben dazu geführt, dass einige Eisenbasiswerkstoffe verfügbar sind, die feinst-/ nanokristallin erstarren. Sie zeichnen sich dadurch aus, dass durch Zugabe von Legierungselementen auch bei relativ geringeren Abkühlraten ein amorphes/nano-/feinstkristallines Gefüge entsteht. Diese Werkstoffe weisen eine hohe Verschleißbeständigkeit, sowie eine extrem geringe Wärmeleitfähigkeit auf, was eine Anwendung sowohl als Verschleißschutz- als auch Wärmedämmschicht möglich erscheinen lässt. Beim Beschichten von Aluminiumlegierungen mit Fe-Basiswerkstoffen stellt die Bildung spröder intermetallischer Phasen $\left(\mathrm{Fe}_{\mathrm{x}} \mathrm{Al}_{\mathrm{y}}\right)$ eine besondere Problematik dar. Der Einsatz der geregelten Lichtbogentechnik ermöglicht jedoch einen definierten Energieeintrag, so dass das Aufschmelzen des Grundwerkstoffes stark reduziert und somit die Bildung intermetallischer Phasen weitgehend unterdrückt werden kann. Zudem scheint diese Verfahrensweise ein hohes Potenzial zu haben, da in Folge des minimalen Energieeintrages in das Substrat hohe Abkühlraten realisiert werden können.

Für einen sicheren Einsatz dieser Werkstoff- und Beschichtungsstrategie in $\mathrm{kmU}$ gibt es derzeit keine abgesicherten Erkenntnisse, die es kmU ermöglichen, neue Arbeitsfelder mit einem kostengünstigen und innovativen Prozess zu erschließen.
Durch den zunehmenden Einsatz von Aluminiumlegierungen treten vermehrt Verschleißprobleme an hoch belasteten Oberflächen auf. So sind beispielsweise Ventilsitze in Verbrennungsmotoren hohem Verschleiß aufgrund von Reibung und hoher Temperaturen ausgesetzt. Zum Verschleißschutz von Aluminiumlegierungen kommen heutzutage unterschiedliche Verfahren, wie Beschichten der Oberflächen oder Einpressen von Inlays, zum Einsatz. Diese können die Lebensdauer der Bauteile z.T. erheblich verlängern.

Das Einpressen von Inlays in die Bauteile ermöglicht es, den Verschleiß, beispielsweise bei Gleitlagern oder Ventilsitzen, zu minimieren. Zum Einsatz kommen dabei Einsätze beispielsweise aus Stahlwerkstoffen oder Keramik [Wan 98, Ano 97]. Die Herstellung solcher Bauteile erfordert jedoch höchste Anforderungen an den Fertigungsprozess. So müssen Übermaßpassungen sowohl beim Inlay als auch beim Bauteil gefertigt werden. Anschließend werden die Einsätze in bzw. auf die Bauteile aufgeschrumpft und/oder gepresst. Bei der Konstruktion ist zu beachten, dass ausreichend Platz für die Einsätze im Bauteil vorgesehen wird und dass durch unterschiedliche Materialien die Wärmeleitung begünstigt bzw. verschlechtert werden kann. Während des Betriebes kann es aufgrund thermischer und mechanischer Beanspruchungen zum Lockern der Inlays kommen.

Eine andere Möglichkeit besteht darin, die Einsätze während des Gießvorganges in das Bauteil zu integrieren. So werden häufig Graugussbuchsen in Pkw Motorblöcke eingegossen um das Reibungs- und Verschleißverhalten der Zylinder zu verbessern [Spa 01, Mai 95]. Jedoch ist auch hier ausreichender Bauraum bei der Konstruktion vorzusehen.

Der Verschleißschutz von Aluminiumlegierungen mittels dünner Schichten, welche beispielsweise mittels PVD oder galvanischen Verfahren aufgetragen werden, ist ebenfalls möglich. Diese bieten trotz geringer Schichtdicken einen guten Verschleißschutz und vermindern die Reibung [Sch 97], [Rib 99]. Jedoch kann es aufgrund unterschiedlicher thermischer Ausdehnungskoeffizienten der Substrat- und Beschichtungswerkstoffe zum Aufbrechen der Beschichtungen, dem sogenannten „Eierschaleneffekt“, kommen [Sen 04].

Thermische Spritzschichten ermöglichen nahezu unbegrenzte Möglichkeiten Substrat- und Schichtwerkstoffe zu kombinieren. Daher werden Aluminiumsubstrate teilweise mit thermisch gespritzten Verschleißschutzschichten versehen. Durch die Optimierung der Legierungszusammensetzung können die Reibwerte und der Verschleiß vermindert und somit die Lebensdauer der Bauteile verlängert werden. Als Beschichtungsverfahren kommen hier beispielsweise das atmosphärische Plasmaspritzen oder das Lichtbogenspritzen zum Einsatz. Unter Berücksichtigung der Innendurchmesser von Zylinderlaufbahnen müssen speziell angepasste Brennersysteme eingesetzt werden [Gad 04, McC 95, Uoz 05]. Jedoch ergeben sich Nachteile für diese Technologie aufgrund der geringen 
Haftfestigkeiten und der relativ großflächigen Anwendung.

Eine Möglichkeit zur Verbesserung der Haftfestigkeiten wäre ein Beschichtungsprozess bei dem eine metallurgische Anbindung erfolgt. Mittels Auftragschweißen lassen sich Schichten mit guten Haftfestigkeiten zum Grundwerkstoff herstellen, da die Substrate lokal aufgeschmolzen werden und eine gemeinsame Schmelze entsteht. Unterschiedliche Schichtsysteme wurden entwickelt, um die Verschleißeigenschaften zu optimieren. Dabei kommen häufig Verbundpulversysteme mit eingelagerten Hartstoffen (z.B. Wolframschmelzkarbide, Vanadiumkarbide oder Titanboride) zum Einsatz. Um wirtschaftlich Beschichtungen mit ausgezeichnetem Verschleißverhalten aufzutragen, sollten Eisenbasiswerkstoffe verwendet werden. Jedoch ergeben sich hieraus metallurgische Schwierigkeiten. Aufgrund der geringen Löslichkeit von Aluminium in Eisen und der Bildung spröder intermetallischer Phasen $\left(\mathrm{Fe}_{\mathrm{x}} \mathrm{Al}_{\mathrm{y}}\right)$ kommt es zu Rissbildung in der Grenzschicht und somit zum Versagen der Verbindung [Wag 01, Rad 97, Zer 98]. Nur wenn es gelingt, die Ausbreitung des Phasensaumes durch eine angepasste Wärmeführung auf unter $5 \mu \mathrm{m}$ zu begrenzen, kann eine solche Verbindung hinreichende Haftzugfestigkeiten erreichen.

Eine Möglichkeit den Wärmeeintrag in den Grundwerkstoff zu minimieren, bietet die Lasertechnologie. Auftragschweißprozesse von Eisenbasislegierungen (FeCrC) auf Aluminiumsubstrate wurden beispielsweise von Iwatani durchgeführt [lwa 05]. Es konnte nachgewiesen werden, dass der Wärmeeintrag beim Auftragschweißen mittels Diodenlaser gegenüber dem Auftragschweißen mittels $\mathrm{CO}_{2}$ Laser verringert werden kann. Dadurch kann der Aufmischungsgrad auf ca. 8\% begrenzt werden. Jedoch ergibt sich ein breiter Saum intermetallischer Phasen was zu Rissen an der Grenzschicht Substrat/ Beschichtung führt.

Aus der Schweiß-/ Löttechnik ist dieses Phänomen bereits hinreichend bekannt. Um das Phasenwachstum zu begrenzen, werden dort speziell angepasste Prozesse angewandt. So kommen geregelte Kurzlichtbogenprozesse zum Einsatz, um den Energieeintrag in die Bauteile zu steuern [Goe 05, Dor 01, Tro 05]. Das ist beispielsweise an dem zeitlichen Leistungsverlauf ersichtlich. In Abb. 1 ist der zeitliche Verlauf der Schweißleistung eines konventionellen dem eines geregelten Kurzlichtbogenprozesses gegenübergestellt.

Beim CMT Prozess (Fronius) wird während der Kurzschlussphase der Schweißstrom minimiert. Um jedoch ein Ablösen des Schweißtropfens zu gewährleisten, wird der Drahtvorschub umgekehrt und der Draht zurückgezogen. Die Neuzündung des Lichtbogens kann somit nahezu leistungsfrei erfolgen. Dieser Vorgang wiederholt sich bei jedem Kurzschluss wobei Frequenzen von bis zu $70 \mathrm{~Hz}$ erreicht werden. Der Gesamtwärmeeintrag ist dabei minimiert und eine Spritzerbildung nahezu ausgeschlossen [Tro 05]. Bei der von EWM entwickelten Anlage erfolgt ein Anste-

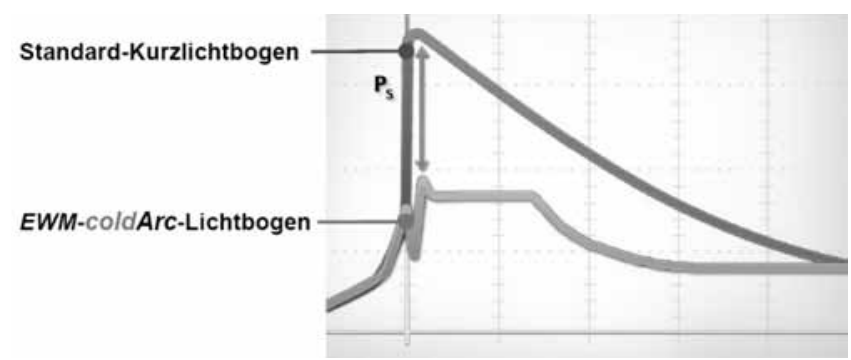

Abb. 1. Leistung beim Wiederzünden des Lichtbogens beim konventionellen und beim geregelten Kurzlichtbogen /www.ewm.de/

Rys. 1. Moc łuku przy ponownym zajarzeniu dla konwencjonalnego i sterowanego łuku zwarciowego (www.ewm.de)

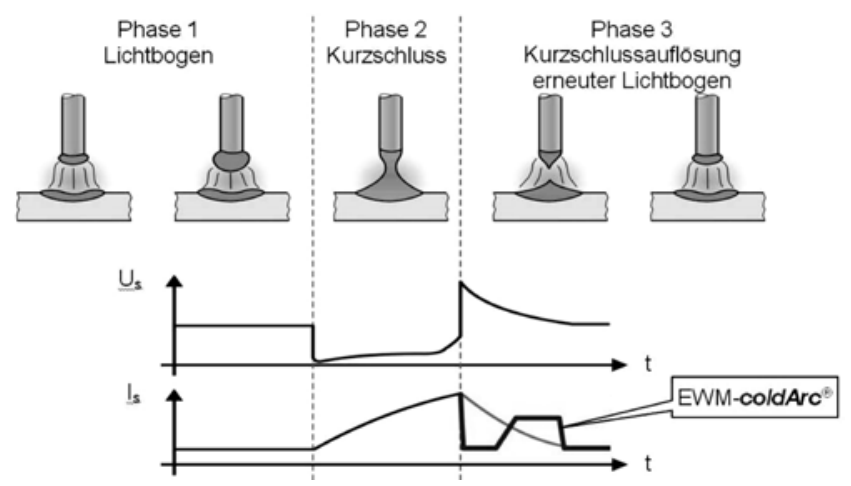

Abb. 2. Zeitlicher Strom- /Spannungsverlauf beim coldArc- und beim konventionellen Kurzlichtbogenschweißen /www.ewm.de/

Rys. 2. Przebieg natężenia prądu i napięcia łuku dla procesu spawania metodą coldArc i konwencjonalnego spawania zwarciowego (www.ewm.de)

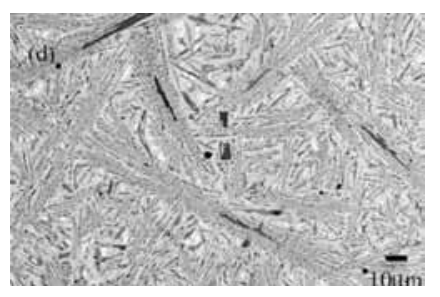

Abb. 3. Nano-/feinstkristallin erstarrter FE-Basis Legierung www.nanosteelco.com/

Rys. 3. Nanokrystaliczna drobnoziarnista struktura stopu na osnowie $\mathrm{Fe}$

igen des Schweißstromes im Kurzschlussfall, wodurch aufgrund des Pinch-Effektes der Tropfen vom Drahtende abgelöst wird. Jedoch muss hierzu der Strom kurz vor Auflösen der Kurzschlussbrücke in einem kurzen Intervall digital heruntergeregelt werden. Der zeitliche Strom- /Spannungsverlauf beim herkömmlichen Kurzlichtbogenschweißen und beim coldArc -Verfahren ist in Abb. 2 dargestellt. Somit wird jeder einzelne Tropfenübergang kontrolliert. Durch diese Regelung wird der Wärmeeintrag in das Substratmaterial minimiert und ein äußerst spritzerarmer Lichtbogenprozess ermöglicht. Untersuchungen zum Fügen von Blechen mit geringerer Stärke sowie zur Bearbeitung zinkhaltiger Zusatzwerkstoffe belegen den Erfolg dieser Technologien [Dor 01, Dor 05, Goe 05]. Weitere Technologien wie STT von Lincoln oder CP von Cloos arbeiten mit ähnlicher Technik. Hier wird ebenfalls der Wärmeeintrag und damit die Spritzerbildung minimiert.

Neueste Entwicklungen in der Werkstofftechnologie haben zu nanokristallin erstarrenden Werkstoffen unter praxisnahen Einsatzbedingungen geführt. Insbesonde- 

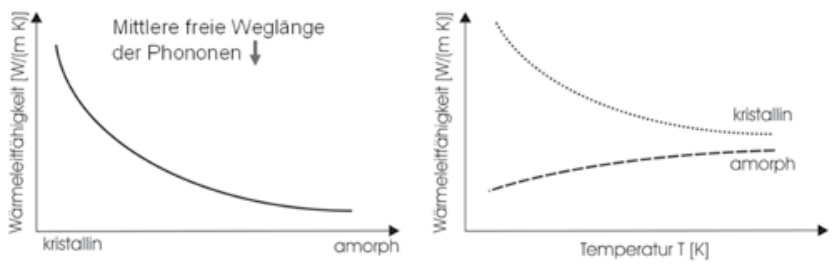

Abb. 4. Verringerung der Wärmeleitfähigkeit metallischer Werkstoffe beim Übergang zu amorpher Mikrostruktur [Nan 97]

Rys. 4. Zmniejszenie przewodności cieplnej materiałów metalicznych przy przejściu do struktury amorficznej [Nan 97]

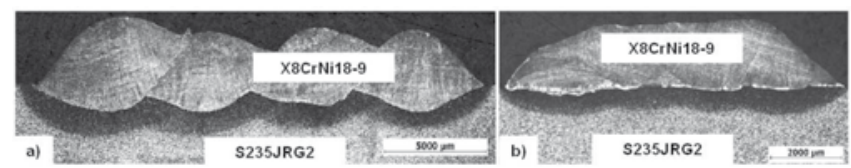

Abb. 5. Verringerter Aufmischungsgrad bei Verwendung der geregelten Kurzlichtbogentechnologie (b) im Vergleich zu konventioneller Sprühlichtbogentechnik (a)

Rys. 5. Zmniejszenie stopnia wymieszania napoin przy zastosowaniu technologii sterowanego łuku zwarciowego (b) w porównaniu z konwencjonalnym łukiem natryskowym (a)

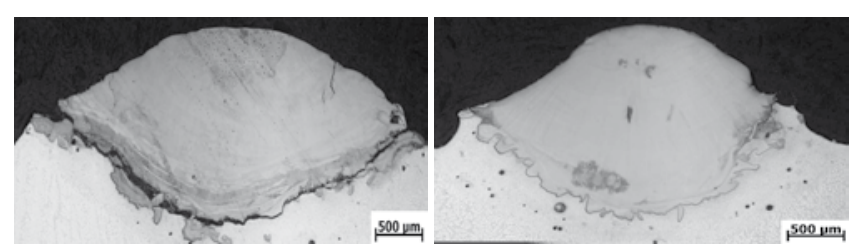

Abb. 6. Beschichtung hergestellt mittels geregelter Kurzlichtbogentechnik mit erhöhtem Wärmeeintrag (links), mit optimiertem Wärmeeintrag (rechts)

Rys. 6. Warstwa wytworzona przez napawanie techniką sterowanego łuku zwarciowego z podwyższoną (z lewej strony) oraz z optymalną (z prawej strony) ilością wprowadzonego ciepła

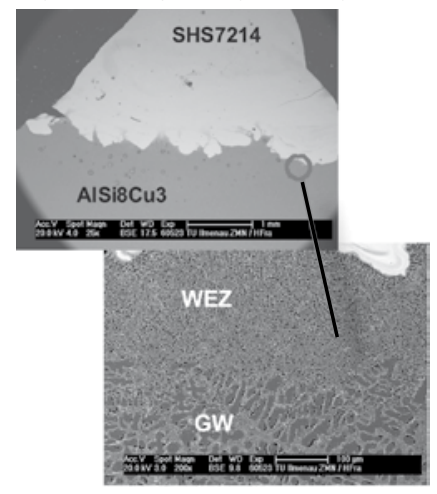

Abb. 7. Beschichtung aus nanokristallin erstarrendem Eisenbasiswerkstoff auf Aluminiumsubstrat.

Rys. 7. Warstwa z nanostruk turalnie krystalizującego materiału na osnowie żelaza nałożona na podłoże ze stopu aluminium

re handelt es sich hierbei um Fe-Basis Legierungen die mit Mo, W, C, Mn, Si und B legiert sind und amorph mit eingelagerten Nanokristalliten erstarren (Abb. 3) [Bra 05, Hof 06].

Die Beschichtungen besitzen gegenüber herkömmlichen Stählen durch inre nanokristalline/amorphe Struktur, die abhängig von den werkstoffspezifischen Prozessbedingungen (z.B. Abkühlrate, Unterkühlung) entstehen kann, erheblich größere Festigkeiten bei ausreichender Bruchdehnung. Ein weiterer durch die Nanokristallinität hervorgerufener Effekt ist eine deutlich im Vergleich zu konventionellen Metallen geringere Wärmeleitfähigkeit, die in der Größenordnung von $\mathrm{ZrO}_{2}$-Keramiken liegt [Hol 03].
Die Wärmeleitfähigkeit in metallischen Festkörpern wird von der Elektron-Elektron- und ElektronStörstellen-Wechselwirkung sowie von der PhononElektron und Phonon-Phonon-Wechselwirkung bestimmt. Bei abnehmender Korngröße wird die Elektron-Elektron-Wechselwirkung zunehmend gestört und die Wärmeleitfähigkeit nimmt ab. Gleichzeitig nimmt die mittlere freie Weglänge der Phononen ab, so dass auch die Wärmeleitfähigkeit über Gitterschwingungen abnimmt. Somit sinkt beim Übergang in nanokristalline/ amorphe Kristallstrukturen die Wärmeleitfähigkeit von metallischen Werkstoffen (Abb. 4) [Hol 03, Nan 97].

Aufgrund der geringen Wärmeleitfähigkeit nanokristallin erstarrender Eisenbasislegierungen ergibt sich für derartige Beschichtungen ein zusätzliches Potenzial für die Verwendung als metallische Wärmedämmschichten [Bra 01, Bra 05, Shi 05].

Der gezielte Wärmeeintrag, der beim Fügen mittels geregelter Kurzlichtbogentechnik ermöglicht ist, ist auch für das Auftragschweißen vorteilhaft. Erste grundlegende Untersuchungen zum Auftragsschweißen mittels geregelter Lichtbogentechnik sind bereits veröffentlicht worden und verdeutlichen, dass Aufmischungsgrade unterhalb $5 \%$ möglich sind /Wil 06/. Im Vergleich konventioneller Sprühlichtbogen - geregelter Kurzlichtbogen (Abb. 5) ist ersichtlich, dass der Energieeintrag in die Grundwerkstoffe wesentlich verringert werden kann.

Gilt es, Aluminiumsubstrate zu beschichten, muss der Energieeintrag in den Grundwerkstoff optimiert werden. Mittels konventioneller Kurzlichtbogentechnik ist das Beschichten von Aluminiumwerkstoffen mit Eisenbasislegierungen, wie X8CrNi18-9, aufgrund des unkontrollierten Wärmeeintrages bei der Lichtbogenneuzündung nicht ermöglicht. Jedoch kann mit geregelter Kurzlichtbogentechnik, wie dem coldArc-Prozess, der Energieeintrag soweit minimiert werden, dass das Wachstum intermetallischer Phasen begrenzt wird. Hierfür muss aber auch beim Beschichten mit geregelter Kurzlichtbogentechnik eine Prozessoptimierung erfolgen. In Abb. 6 - links ist eine Beschichtung abgebildet, welche mittels geregelter Kurzlichtbogentechnologie mit nicht optimiertem Energieeintrag aufgrtragen wurde. Es ist ersichtlich, dass es, aufgrund des hohen Energieeintrages und der damit verbundenen Sprödphasenbildung, zu Rissbildung in der Grenzschicht zwischen Substrat und Beschichtung kommt. Beim Beschichten mit minimiertem Energieeintrag hingegen (Abb. 6 - rechts) kann die Phasenbildung begrenzt werden, sodass keine Rissbildung auftritt.

Die Machbarkeit des Auftragschweißens von nanokristallin erstarrenden Eisenbasiswerkstoffen auf Aluminiumsubstraten mittels geregelter Kurzlichtbogentechnologie wurde in grundlegenden Untersuchungen nachgewiesen [Wil 07]. Während der Untersuchungen kamen sowohl die CMT- als auch die coldArc-Technologie zum Einsatz. In Abb. 7 ist eine Auftragschweißung von SHS7214 auf einer Aluminiumgusslegierung 


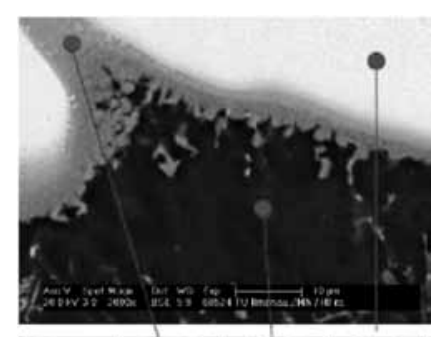

\begin{tabular}{|c|c|c|c|}
\hline Element & Wt-\% & Wt.\% & Wt-\% \\
\hline $\mathrm{Mg}$ & - & 4,4 & - \\
\hline $\mathrm{Al}$ & 58,1 & 72,4 & - \\
\hline $\mathrm{Si}$ & 12,9 & 20,9 & 1,3 \\
\hline $\mathrm{Mo}$ & - & - & 1,1 \\
\hline $\mathrm{Cr}$ & 4,5 & - & 21,8 \\
\hline $\mathrm{Fe}$ & 18,7 & - & 72,1 \\
\hline $\mathrm{Cu}$ & 3,9 & - & - \\
\hline $\mathrm{W}$ & 1,9 & 2,3 & 3,7 \\
\hline
\end{tabular}

Abb. 8. Ausbildung eines intermetallischen Phasensaums $<5 \mu \mathrm{m}$

Rys. 8. Tworzenie się faz międzymetalicznych o wielkości $<5 \mu \mathrm{m}$
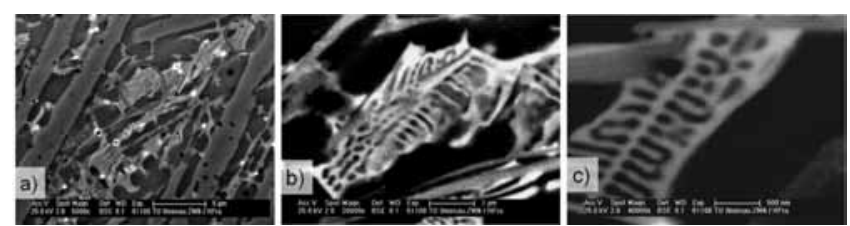

Abb. 9. Nanokristallin erstarrtes Gefüge des Auftragschweißwerkstoffes bei hinreichenden Abkühlbedingungen

Rys. 9. Widok nanostrukturalnie krystalizujących struktur napoin przy różnych warunkach ich układania

dargestellt, welche mit der coldArc-Technologie hergestellt wurde. In der Vergrößerung ist zu erkennen, dass der wärmebeeinflusste Bereich im Aluminium nur ca. $0,2 \div 0,3 \mathrm{~mm}$ beträgt.

Es ist anhand der Ergebnisse ersichtlich, dass der Wärmeeintrag in den Substratwerkstoff minimiert werden kann. Durch eine angepasste Prozessführung lässt sich die Bildung eines spröden Saumes intermetallischer Phasen auf unter $5 \mu \mathrm{m}$ begrenzen (Abb. 8). Wie die EDX-Analysen verdeutlichen erfolgt nahezu keine Aufmischung mit dem Grundwerkstoff. Ca. $10 \mu \mathrm{m}$ neben dem Interface Schicht/Grundwerkstoff ist in der Schicht kein Al und in dem Grundwerkstoff kein $\mathrm{Fe} \mathrm{zu}$ analysieren. Das Interface selber wird aus einem ca. $2 \mu \mathrm{m}$ breiten Phasensaum gebildet.

Durch die hohe Wärmeleitfähigkeit des Aluminiums wird die zugeführte Wärme schnell abgeleitet, sodass günstige Bedingungen für eine nanokristalline Erstarrung vorliegen. In Abb. 9 ist das Gefüge des Auftragwerkstoffes dargestellt. Zu erkennen sind Strukturen deren Abmessungen deutlich unter $1 \mu \mathrm{m}$ betragen. Aufgrund der feinstkristallinen Erstarrung ergeben sich Härtewerte von $1008 \pm 112$ HV0,01.

\section{Vorgehensweise}

Mit dem Einsatz der geregelten Kurzlichtbogentechnik werden zwei Ziele verfolgt. Einerseits gilt es, ein kostengünstiges Verfahren für das Auftragen hochwertiger Beschichtungen anzubieten und andererseits sollen Aluminiumsubstrate mittels Auftragschweißens von Eisenbasislegierungen beschichtet werden. Insbesondere kann durch den geregelten MIG-Lichtbogen beim Beschichten von Aluminiumsubstraten eine „Reinigung" und Aktivierung des Grundwerkstoffes erzielt werden, so dass günstige Bedingungen für die Benetzung vorliegen. Darüber hinaus führen die Abkühlraten dazu, dass einerseits der aufzutragende Fe-Basiswerkstoff amorph/nanokristallin erstarrt und die Bildung intermetallischer Phasen weitgehend unterdrückt werden kann. Weiterhin ergibt sich eine geringe Aufmischung, wodurch die Eigenschaften des Schichtwerkstoffes nahezu erhalten bleiben.

Erste grundlegende Untersuchungen werden an linienförmigen Auftragraupen auf einer AISi-Gusslegierung mit einem Fülldraht auf $\mathrm{Fe}-\mathrm{Basis}(\mathrm{Fe} \mathrm{Cr} \mathrm{Mo}$ W Ti B C Si Mn) durchgeführt. Dabei sollen grundlegende Erkenntnisse zur Prozessführung (zeitlicher Strom-/Spannungsverlauf, Drahtvorschub, Schweißgeschwindigkeit), sowie über die einzustellenden Prozessbedingungen zum Erlangen einer nanokristallinen Erstarrung erarbeitet werden. Das Prozessfenster wird basierend auf den bereits beim Antragsteller vorliegenden empirischen Erkenntnissen systematisch erarbeitet, um zu einer direkten Korrelation der Prozessbedingungen und der Legierungszusammensetzung mit den herrschenden Erstarrungsbedingungen und Werkstoffeigenschaften zu gelangen. Anhand von Temperaturmessungen im Prozess werden der Zeit-Temperatur-Verlauf und somit auch die für die Erstarrungsmorphologie wesentlichen $\mathrm{Ab}$ kühlbedingungen abgeleitet. In weiteren Untersuchungen sollen in Abstimmung mit den Mitgliedern des pbA Beschichtungen auf verschiedenen Al-Legierungen hergestellt werden. Dabei sollen neben Gusslegierungen unterschiedlicher Zusammensetzung auch Knetlegierungen (z.B. 5xxx und 7xxx) zum Einsatz kommen. Die Abkühlrate wird jedoch nicht nur vom Werkstoff, sondern auch von der Geometrie des Substrates bestimmt, so dass auch dieser Einfluss Gegenstand der Untersuchungen ist. Im Hinblick auf den Ersatz von Inlay-Dichtsitzen werden vor allem kreisförmige Beschichtungen hergestellt, für die es Slope-in und Slope-out Strategien zu entwickeln gilt. Die Schichten werden licht- und rasterelektronenmikroskopisch untersucht, darüber hinaus erfolgt eine hochaufgelöste Analyse der entstandenen Phasen

\section{Ergebnisse}

Ausgehend von den Voruntersuchungen erfolgten umfangreiche Schweißversuche mit den in Bild 10 dargestellten Fülldrähten.

Auf Grund der Rissbildungsproblematik wurden zum Auftragschweißen 2 unterschiedliche 


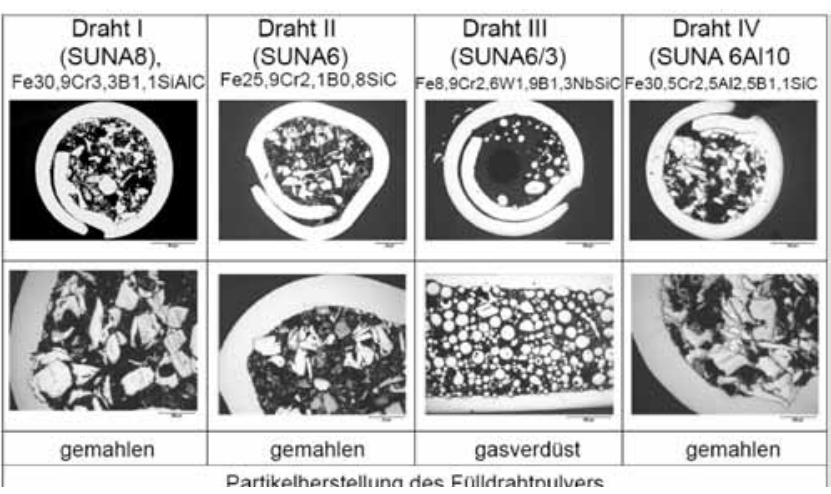

Abb. 10. Fülldrahtzusammensetzungen und Drahtquerschliffe Rys. 10. Skład chemiczny i przekrój drutów proszkowych

\section{Energiereduzierter AC-Impulslichtbogen, MSG Pulse CP}

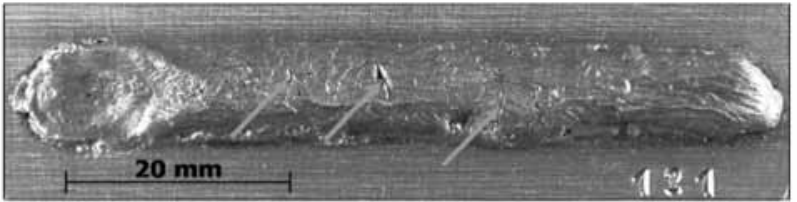

Schweißparameter:

$v_{\mathrm{S}}=0,65 \mathrm{~m} / \mathrm{min}$

$\mathrm{v}_{\mathrm{D}}=4,90 \mathrm{~m} / \mathrm{min}$

Pulszeit $=2,30 \mathrm{~ms}$

Grundstrom $=80 \mathrm{~A}$

Negativer Grundstrom $=130 \mathrm{~A}$

Negative Spannung $=10 \mathrm{~V}$

Energiereduzierter Kurzlichtbogen, ColdArc, mit WIG-Vorwärmung
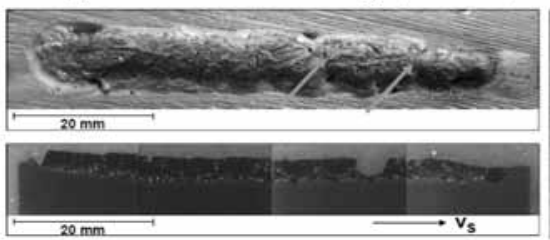

Schweißparameter: Substrat: AC-AlSigCu3

$v_{\mathrm{S}}=0,50 \mathrm{~m} / \mathrm{min} \quad$ Schutzgas $=$ Argon $4.6 \mathrm{mit} 22 \mathrm{l} / \mathrm{min}$

$V_{D}=3.50 \mathrm{~m} / \mathrm{min} \quad$ Auftragwerkstoff: Draht I, Fe30,9Cr3,3B1,1Si0,1Al0,1C

$\mathrm{U}_{\mathrm{S}}=25,0 \mathrm{~V}$

WIG-Parameter:

Stromstärke $=200 \mathrm{~A}$, Frequenz $=100 \mathrm{~Hz}$

AC-t-Balance $=40 \%$

Abb. 11. Aufsicht auf Auftragschweißungen mit einem energiereduzierten AC-Impulslichtbogen (nach oben) und einem energiereduzierten Kurzlichtbogen mit WIG-Vorwärmung

Rys. 11. Widok napoin napawanych łukiem impulsowym AC o zredukowanej energii (u góry) i łukiem zwarciowym o zredukowanej energii z podgrzewaniem metodą TIG
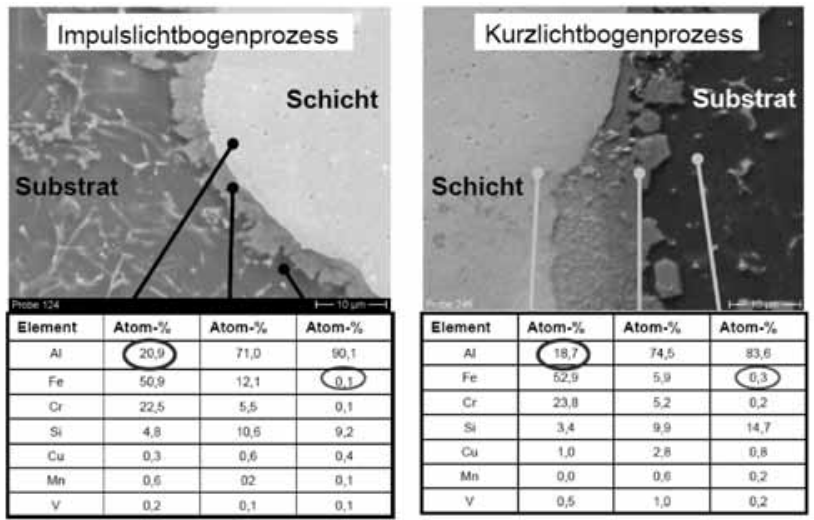

Abb. 12. Querschliffe und Elementanalysen „optimierter" Auftragschweißungen

Rys. 12. Mikrostruktura i analiza punktowa optymalnych napoin

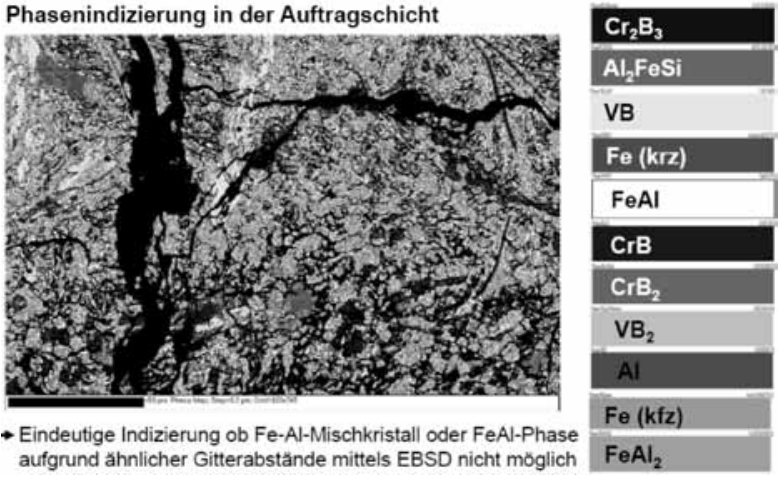

Abb. 13. EBSD-Analyse einer Auftragschweißung Rys. 13. Analiza ESBD napoin

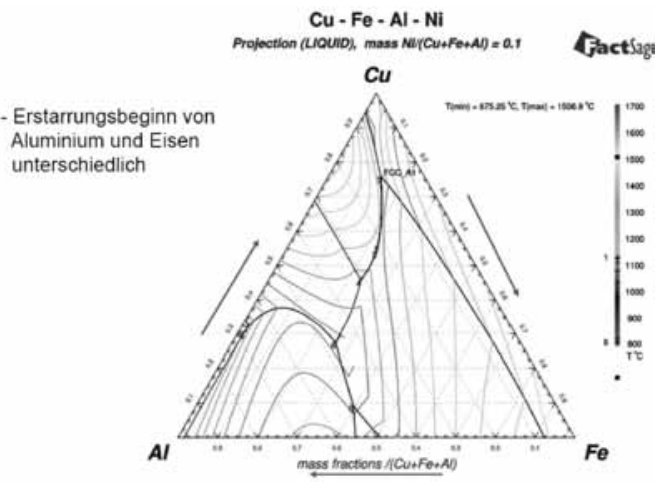

Abb. 14. Berechnetes Phasendiagramm des Systems Cu-Fe-Al-10Ni Rys. 14. Obliczeniowy równowagowy układ fazowy Cu-Fe-Al.-10Ni

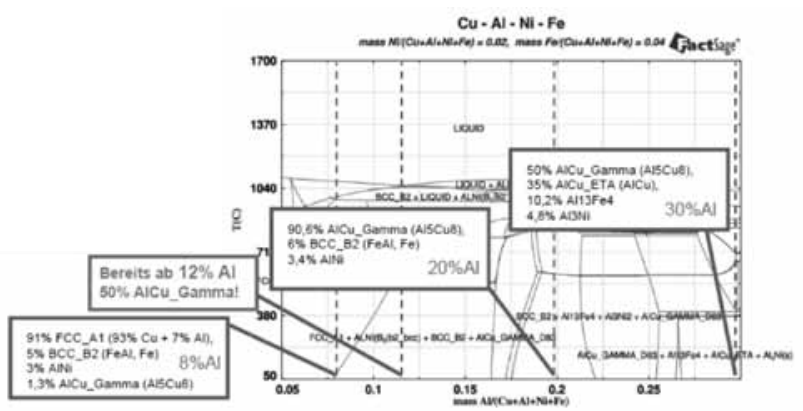

Abb. 15. Berechnete Phasenfelder in Abhängigkeit von der Al-Konzentration

Rys. 15. Obliczone obszary fazowe w zależności od zawartości Al

Prozessstrategien verfolgt. Zum einen kam ein energiereduzierter Kurzlichtbogen mit WIG-Vorwärmung und ein energiereduzierter Impulslichtbogen zum Einsatz. Im Ergebnis muss festgehalten werden, dass ein rissfreies Auftragschweißen mit diesen Prozessen und den o.g. Fülldrähten trotz umfasssender Parameteroptimierung nicht möglich war (ABB: 11).

In Abbildung 12 sind Querschliffe durch die „optimierten" Auftragschweißungen dargestellt. Auffällig ist, dass im Gegensatz zu den Ergebnissen der Vorversuche unabhängig von der gewählten Prozessstrategie ein recht hoher Aufmischungsgrad mit ca. 20\% vorhanden ist. 
Mit dem Ziel, die Auftragschweißwerkstoffe für das Beschichten von Aluminium weiter zu entwickeln, erfolgten umfangreiche Phasenanalysen in den Beschichtungen. Mit den in Abbildung 13 dargestellten EBSD Ergebnissen konnte allerdings keine Korrelation zwischen Rissbildung und Phasenausbildung oder anordnung abgeleitet werden. Als Lösungsansatz wurde das Einbringen von Pufferschichten gewählt.

Mittels Phasendiagrammberechnungen erfolgte die Auslegung der Pufferschicht im System Cu-Fe-Al$\mathrm{Ni}$ (Abb. 14, 15). Aus den Berechnungen geht hervor, dass die Aufmischung unter $12 \%$ Al gehalten werden muss, um intermetallische Phasen zu vermeiden.

Mit der Legierung AlBz5Ni2 gelang es mittels energiereduziertem Impulslichtbogen rissfreie Kreisnähte zu erzeugen (Abb. 16)

Die phasentheoretischen Betrachtungen erfolgten in der weiteren Folge auch für die Entwicklung von Eisenbasislegierungen zum direkten Auftragschweißen sowie einer erforderlichen Temperaturführung. In Abb. 17 sind die Anteile an Fe-Mischkristall und Karbiden in Abhängigkeit von unterschiedlichen Legierungszusammensetzungen dargestellt. In den $\mathrm{Be}$ rechnungen wurde eine Aufmischung von $20 \%$ Al berücksichtigt.
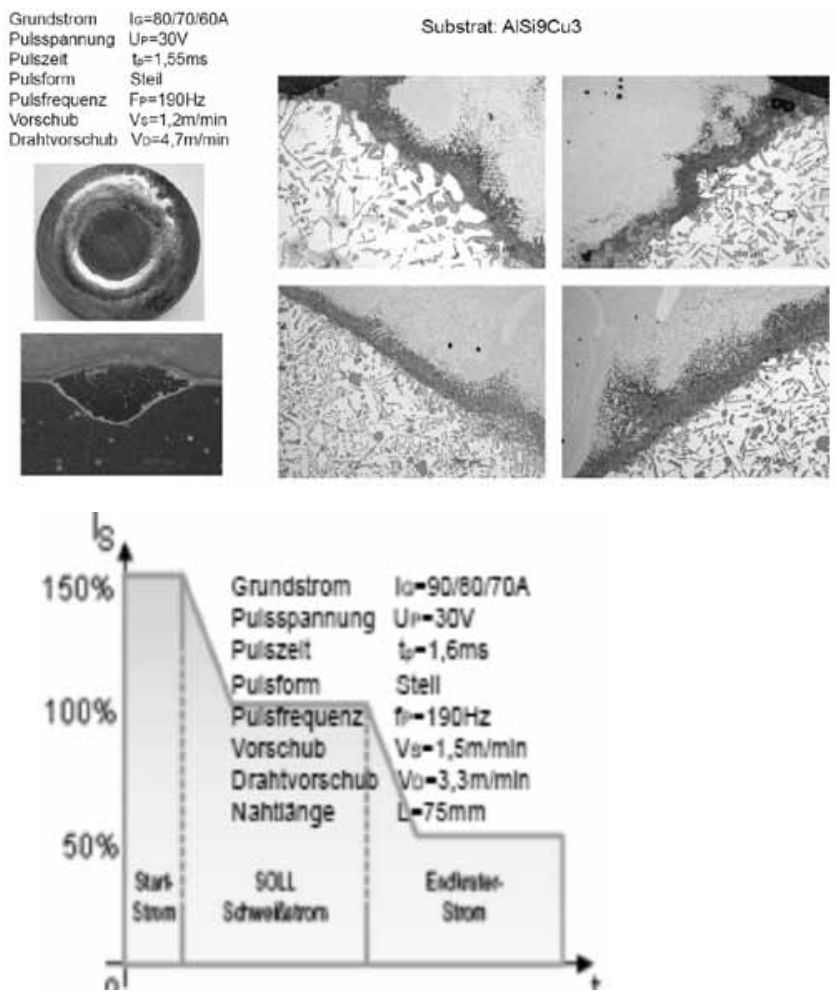

Abb. 16. Auftragschweißung mit dem Pufferwerkstoff AlBz5Ni2 (links) und Prozessführung zur Vermeidung Nahtanfangs- und -endfehlern

Rys. 16. Napawanie z materiałem buforowym AIBZ5Ni2 i sterowanie procesu w celu zmniejszenia wad na początku i końcu napoiny
Berechnete karbidischer Phasen

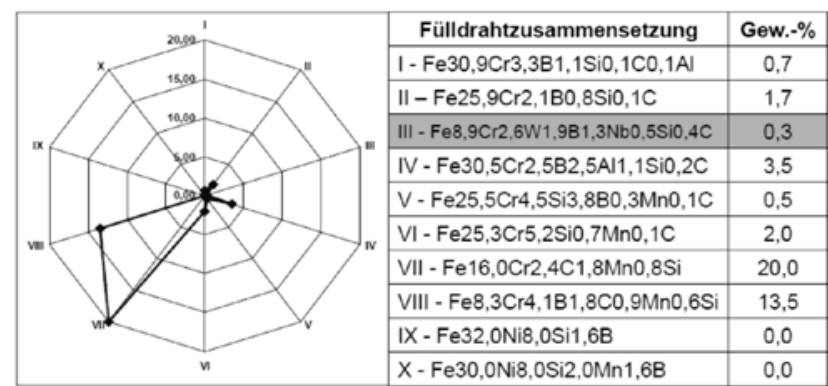

Alle Angaben in Gew. $\%$

Berechnete Fe-Mischkristallanteil

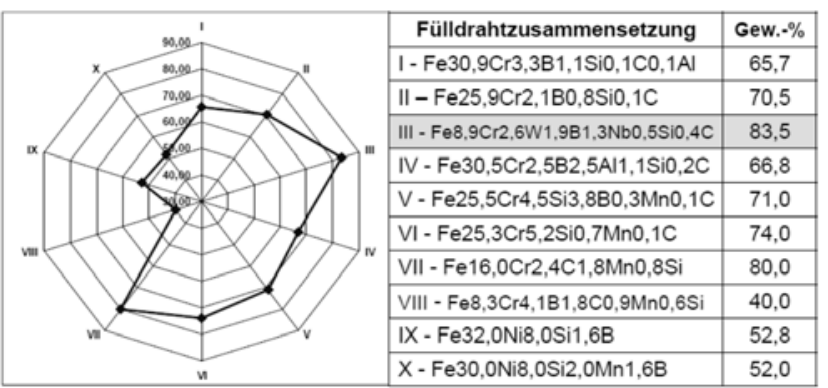

Alle Angaben in Gew.-\%

Abb. 17. Berechnete Gefügebestandteile in Abhängigkeit von der Legierungszusammensetzung bei einer Aufmischung von 20\% Al Rys. 17. Obliczeniowy równowagowy układ przy wymieszaniu z $20 \%$ Al

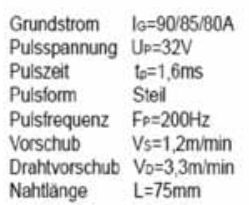

Nahtlànge $\quad L=75 \mathrm{~mm}$
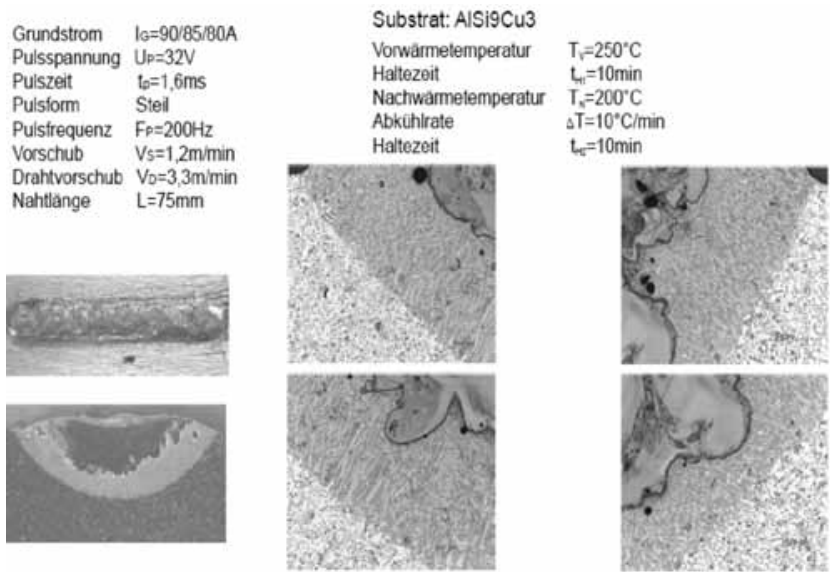

Abb. 18. Auftragschweißung ohne Pufferschicht mit Vor- und $\mathrm{Na}$ chwärmen

Rys. 18. Widok i struktury napoiny bez warstwy buforowej z podgrzaniem przed i po procesie napawania

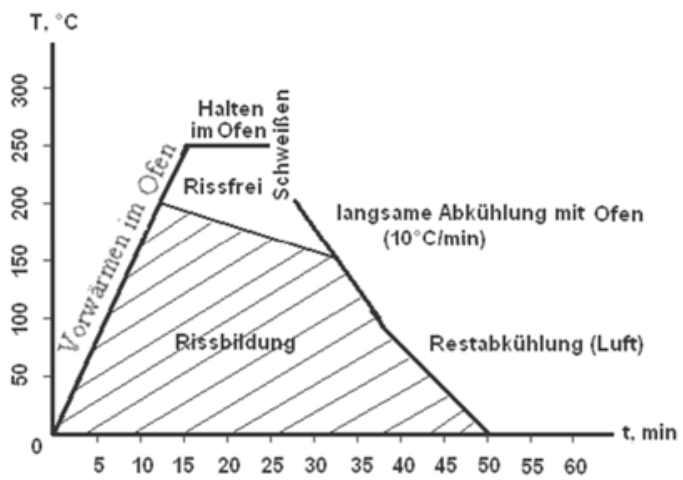

Abb. 19. Temperaturführung zum rissfreien Auftragschweißen Rys. 19. Przebieg temperatury dla wykonywania napoin bez wystąpienia pęknięć 


\section{Zusammenfassung}

Das Auftragschweißen von Fe-Basislegierungen auf Al-Gusswerkstoffe ist auf Grund der Bildung intermetallischer Phasen prozess- und legierungstechnisch problematisch. Es wurden zwei unterschiedliche Prozessstrategien erarbeitet. Zum einen ist das Einbringen einer Al-Bronze eine Lösungsvariante und zum anderen das Einhalten einer definierten Temperaturführung bei der der Werkstoff zunächst vorgewärmt und nach dem Schweißprozess nachgewärmt werden muss. Zur gezielten Legierungs- und Prozessentwicklung wurden phasentheoretische Berechnungen eingesetzt, um abschätzen zu können, welche Phasen sich in Abhängigkeit von der Aufmischung ausbilden.

Mit beiden Prozessstrategien ist das prozesssichere Auftragschweißen von Fe-Basislegierungen auf Al-Gusslegierungen möglich, so dass auf der Basis der dargestellten Ergebnisse Eisenbasisbeschichtungen auf unterschiedlichen
Aluminiumlegierungen kostengünstig herstellbar sind bzw. mit geringem experimentellem Aufwand die ausgearbeiteten Prozessstrategien auf die jeweilige Anwendung zu projiziert werden kann. Die wirtschaftlichen Vorteile bestehen vor allem in der leichten Handhabbarkeit, der hohen Energieeffizienz und den damit verbundenen niedrigen Prozesskosten. So ist beispielsweise bei der Herstellung von Ventilsitzen eine Verkürzung der Fertigungsfolge möglich. Bisher wurden sowohl die Al-Guss als auch die Inlays mit Passungen versehen, welche in weiteren Prozessschritten aufgeschrumpft und/oder eingepresst wurden. Mit der Bereitstellung einer Technologie zum Beschichten der Aluminiumbauteile kann die Fertigungsfolge auf Beschichten und Endbearbeiten reduziert werden. Weiterhin ergeben sich Vorteile aufgrund verlängerter Lebensdauer und somit verringerter Instand-haltungskosten.

\section{Literaturverzeichnis}

[Ano 97] Anonym; Yamaha verbessert Design des Ventilsitzes durch neues Verbindungsverfahren; Metal Powder Report; Band 52 1997; Heft 10; Seite 16-17.

[Bra 01] Branagan D. J.; Swank W. D.; Haggard D. C.; Fincke J.R.; Wear-Resistant Amorphous and Nanocomposite Steel Coatings, Metalurg. and Mater. Trans. A, 32A, (2001), 2615-2621.

[Bra 05] Branagan D.J.; Marshall M.C.; Meacham B.E.; Formation of nanoscale composite coatings via HVOF and wire-arc spraying; ITSC May 2-4 2005 Basel, S. 908-913.

[Dor 01] Dorn L.; Goecke S.-F.; Advantages of reduced heat input during ChopArc-welding and brazing for coated and combined metals for light weight vehicles, DVMTag 2001, Fuegetechnik im Automobilbau, May 2001, Berlin.

[Dor 05] Dorn L.; Goecke S.-F.; ChopArc - MSG Lichtbogenschweißen für den Ultraleichtbau, Fraunhofer IRB Verlag, 2005, A3-A16, A21-A23.

[Gad 04] Gadow R.; Robotic thermal spray process coats cylinder bores in engines; Advanced Materials \& Processes; Band 162; 2004; Heft 8; Seite 59-60.

[Goe 05] Goecke S. F.; Energiereduziertes Lichtbogen-Fügeverfahren für wärmeempfindliche Werkstoffe,DVSBerichte Band 237; pp. 43-48.

[Hof 06] Hoffmeister H.-W.; Schnell C.: Leichtmetallkurbelgehäuse mit nanokristalliner Spritzschicht. In VDI Berichte 1906, 237-246, (2006).

[Hol 03] Holister P.; Vas C. R.; Harper T.; Nanocrystalline Materials, Technologie White Papers nr. 4; Cientifica, Ltd. (2003).

[Iwa 05] Iwatani S.; Ogata Y.; Uenishi K.; Kobayashi K.F.; Tsuboi A.; Laser cladding of $\mathrm{Fe}-\mathrm{Cr}-\mathrm{C}$ alloys on A5052 aluminum alloy using diode laser; Materials Transactions, JIM Band 46; 2005; Heft 6; Seite 1341-1347.

[McC 95] McCune, R.C.; Thermal spraying of cylinder bore sur- faces for aluminium engine blocks; Welding Journal, New York; Band 74; 1995; Heft 8; Seite 41-47.

[Mai 95] Maier K.; Zylinderlaufflächen im modernen Motorenbau; Ulmer Gespräch, 17, 1995; Seite 44-50.

[Man] Mantel W.; Die MIG- Schweißung von Al-Legierungen; Linde AG Werksgruppe München, Schweißtechnik- Hinweise für die Praxis $X$ 65/1.

[Nan 97] Nan C-W.; Birringer R.; Clarke D.R.; Gleiter H.; Effective thermal conductivity of particulate composites with interfacial thermal resistance; J. Appl. Phys. 81; 10, 15 May 1997.

[Uoz 05] Uozato S.; Nakata K.; Ushio M.; Evaluation of ferrous powder thermal spray coatings on diesel engine cylinder bores; Surface and Coatings Technology Band 200, 2005; Heft 7; Seite 2580-2586.

[Rad 95] Radscheid C.; Schubert E.; Sepold G.; Laserstrahlfügen von Werkstoffkombinationen am Beispiel von AluminiumStahl-Verbindungen; Präzisionsbearbeitung mit Festkörperlasern, 1996.

[Rad 97] Radscheit C.; Laserstrahlfügen von Aluminium mit Stahl. Dissertation Universität Bremen 1996; Bremen: Verlag BIAS; 1997.

[Rib 99] Ribeiro C.; PVD-Beschichtung von Aluminiumlegierungen; Ingenieur-Werkstoffe Band 8; 1999; Heft 1; Seite 4244.

[Sch 97] Scholten F.U.; Funktionelle Überzüge veredeln aus Alu-Bauteil. Oberflächenschichten erweitern die Einsatzmöglichkeiten; Quelle Industrieanzeiger Band 119; 1997; Heft 41; Seite 50-52.

[Sen 04] Senf J., Berg G., Friedrich C., Broszeit E., Berger C., Stippich F., Engel P., Wolf G.K.: Beanspruchungsverhalten von PVD-CrN Beschichtungen auf Leichtmetallwerkstoffen; Materialwissenschaft und Werkstofftechnik; Volume 29, Issue 1, Pages 9 - 15; 2004.

[Shi 05] Shin, D.; Gitzhofer, F.; Moreau, C.; Development of metal based thermal barrier coatings (MBTBCs) for low heat rejection diesel engines; ITSC May 2-4 2005 Basel, S. 915-919. 
[Spa 01] Spangenberg S.; Schuller G.; Grauguß-Laufbuchsen - Lösung für extreme Anforderungen bei Benzin- und Dieselanwendungen; VDI-Berichte; Band 1612; 2001 Seite 77-82; Düsseldorf: VDI-Verlag.

[Tro 05] Trommer G.; CMT-Schweißprozess in der Praxis; Fronius International $\mathrm{GmbH}$.

[Tro 06] Trommer G.; Dünne Blechbauteile automatisch gut gefügt. Lichtbogenschweissen.; Blech InForm, Heft 4, Seite 66-69, 2006.

[Wag 01] Wagner F.; Zerner I.; Kreimeyer M.; Sepold G.; Phasenbildung beim Laserstrahlfügen von $\mathrm{Fe} /$ Al-Verbindungen; DVS-Berichte; Band 212; 2001 . Seite 93-98

[Wan 98] Wang Y.S.; Narasimhan S.; Larson J.M.; Schaefer, S.K.; Wear and wear mechanism simulation of he- avy-duty engine intake valve and seat inserts; Journal of Materials Engineering and Performance, Band 7 1998; Heft 1; Seite 53-65.

[Wil 06] Wilden J.; Bergmann J.P.; Dolles M.; Muether R.; Goecke S.-F.; Cladding with new short arc devices, ITSC 2006, Seattle, USA.

[Wil 07] Wilden J.; Bergmann J.P.; Reich S.; Schlichting S.; Schnick T.; Cladding of Aluminum Substrates with Nano Crystalline Solidifying Wear Resistant Iron-Based Materials; ITSC 2007; Beijing, China.

[Zer 98] Zerner I.; Schubert E.; Sepold G.; Laserstrahlfügen von Aluminium mit Stahl. Proceedings "Neues zum Schweißen von Leichtbauwerkstoffen"; Tagungsreihe Dünnblechverarbeitung, Erding; 1998; S. 221-230.

\section{Danksagung}

Die Untersuchungen wurden aus Haushaltsmitteln des Bundesministeriums für Wirtschaft und Technologie (BMWi) über die Arbeitsgemeinschaft industrieller Forschungsvereinigungen „Otto von Guericke" e.V. gefördert und von der Forschungsvereinigung „Schweißen und verwandte Verfahren“ e. V. des DVS unterstützt. Für diese Förderung und Unterstützung sei herzlich gedankt.

\section{Nowości techniczne}

\section{Okulary ochronne od 3M - moda na bezpieczeństwo}

Firma 3M Poland, oddział światowego lidera w dziedzinie innowacji, prezentuje szeroka gamę okularów i gogli ochronnych. Oferta obejmuje m.in. okulary przeciwsłoneczne, okulary do strzelania czy okulary z wymiennymi soczewkami. Wszystkie produkty $3 \mathrm{M}$ z zakresu ochrony wzroku charakteryzuje nie tylko niezawodna jakość, ale również nowoczesny design.

Stylowa jakość, która wyróżnia ofertę okularów ochronnych 3M Poland, to wysoka jakość i gwarancja pełnej ochrony ale również modne kształty i elegancki styl. Większość modeli wyposażono w regulowane elementy, a ich powierzchnie stykające się ze skórą wykonano z miękkich materiałów. Dzięki temu zwiększyła się powierzchnia dopasowania okularów do twarzy, a komfort użytkowania znacząco się poprawił.

Jednym z priorytetów firmy 3M jest zapewnienie naszym klientom bezpieczeństwa w pracy, w domu i w życiu codziennym. Wierzymy bowiem, że zdrowie jest najważniejsze, a większa wygoda poprawia ochronę pracownika, zwiększa jego wydajność i zapewnia zgodność z przepisami. Nasza oferta okularów i gogli ochronnych zapewnia nie tylko funkcje ochronne, ale również elegancki wygląd - mówi Marta Kapała, starszy koordynator marketingu w dziale Bezpieczeństwa Pracy w 3M Poland. - Większość okularów i gogli od 3M chroni również przed promieniowaniem UV oraz została zatwierdzona do użytku zgodnie z normą PN-EN166 i ma oznaczenie CE - dodaje Marta Kapała.

Z myślą o osobach użytkujących sprzęt ochrony wzroku w połączeniu z innym sprzętem ochrony indywidualnej firma 3M Poland poszerzyła swoją ofertę okularów o dwie nowe serie: Tora ${ }^{\mathrm{TM}}$ CCS i Virtua ${ }^{\mathrm{TM}}$ AP. Dla przykładu. Okulary Tora ${ }^{\mathrm{TM}}$ CCS mają zintegrowany System $3 \mathrm{M}^{\mathrm{TM}}$ Cord Control (CCS) pozwalający użytkownikowi na dołączenie wkładek przeciwhałasowych na sznurku, a tym samym jednoczesne wykorzystanie ochronników wzroku i słuchu. Seria Virtua ${ }^{\mathrm{TM}}$ AP ma z kolei ochronny pasek na linii brwi, który chroni oczy przed ewentualnymi odłamkami. W przypadku tego modelu dostępne są różne kolory soczewek.

\section{Coś dla Pań i Panów}

Dla kobiet, których praca wymaga korzystania z okularów ochronnych, firma 3M przygotowała serię Refine 300. Te modne okulary zaprojektowano w taki sposób, aby lepiej przylegały do głowy i twarzy użytkowniczek. Z kolei dla panów, zwracających uwagę na nowoczesny design, firma wprowadziła okulary $3 \mathrm{M}^{\mathrm{TM}}$ Marcus Grönholm, sygnowane przez znanego kierowcę rajdowego. Mają one oprawki z hipoalergicznego metalu z regulowanym noskiem, plastyczne końcówki zauszników zwiększające możliwość regulacji oraz soczewki pokryte powłoką AS-AF zabezpieczające okulary przed zarysowaniem i zaparowaniem. 